\title{
Intraparenchymal Meningioma
}

\author{
Raul Ramirez-Grueso a, c, Sergio I. Patino-Ladino a, Jorge A. Amortegui-Beltran ${ }^{\text {a }}$, \\ Jose Luis Rios ${ }^{\mathrm{a}}$, Lina Estrada-Duque ${ }^{\mathrm{b}}$, Jaime Arias ${ }^{\mathrm{a}}$
}

\begin{abstract}
Meningiomas have been described as the most frequent extra-axial tumor in the brain. Most are benign and correspond to World Health Organization (WHO) grade I; however, there are some reports of cases that shows atypical locations, like subcortical intra-axial meningiomas. This represents a challenge in radiological diagnosis because it could be taken as a metastasis tumor or vascular malformations. The authors bring a case report of a previously healthy patient who presents a traumatic brain injury with no traumatic lesions. A computed tomography (CT) evidenced a left frontal rounded subcortical lesion surrounded by large vasogenic edema. The first diagnostic impression was a metastasis tumor. During surgery, a total resection was completed, and the appearance of the tumor was meningioma. This was later confirmed by histological analysis. The literature was reviewed in order to determine the importance of including intraparenchymal meningiomas in the differential diagnosis of intracranial lesions.
\end{abstract}

Keywords: Meningioma; Intraparenchymal meningioma; Intra-axial meningioma

\section{Introduction}

Meningiomas by definition are benign tumors dependent on the cerebral meninges, specifically on the arachnoid or meningothelial cells [1-4], showing extra-axial location [3]. They constitute one of the most common primary neoplasms of the central nervous system, taking the first or second place in frequency at the intracranial level [5]. Sometimes they have an atypical presentation that lacks dural adhesion $[1-3,6]$, so much so that it has been rarely reported in the literature $[4,5,7,8]$.

Meningiomas normally exhibit typical radiological char-

Manuscript submitted September 22, 2020, accepted October 9, 2020

Published online November 18, 2020

aDepartment of Neurosurgery, Universidad Nacional de Colombia, USS Tunal, Bogota, Colombia

bepartment of Neurosurgery, Universidad Militar Nueva Granada, USS Tunal, Bogota, Colombia

${ }^{\mathrm{c} C}$ Corresponding Author: Raul Ramirez-Grueso, Department of Neurosurgery, Universidad Nacional de Colombia, USS Tunal, Bogota, Colombia.

Email: raulrg0328@hotmail.com

doi: https://doi.org/10.14740/jmc3592 acteristics that facilitate their pre-surgical diagnosis without the need for invasive procedures [1]. But it is precisely the absence of dural adhesion that makes its presurgical diagnostic approach challenging $[4,7,8]$ tipping the scales toward other diagnostic options such as metastases [8], as the case presented in the clinical picture discussed in this article.

\section{Case Report}

A 70-year-old female consulted for 2-year evolution symptoms consisting of syncopal episodes, with loss of postural tone and without the presence of abnormal movements, relaxation of sphincters or other associated symptoms. She referred a frequency of approximately two to three episodes per year without consultation to the emergency services or previous study of the episodes. On the admission day to the institution, she presented an event of the same characteristics with mild traumatic brain injury (TBI) and facial trauma secondary to falling from her own height. For this reason, a simple skull computed tomography (CT) is carried out and a neurosurgery concept is requested.

During the initial evaluation, the patient is found in a Glasgow Coma Scale (GCS) as follows: GCS of 15, acceptable general condition, with vital signs within normal limits, with craniofacial trauma stigmas, bradypsychic, cranial nerves without alterations, preserved muscle strength in all 4 limbs, without sensory deficits and without the presence of pathological reflexes.

Significant vasogenic edema in the extension of the left frontal lobe with volume effect and deviation of the midline to the right is evidenced on the skull CT (Fig. 1). Findings highly suggestive of tumor lesion without clear limits in it were considered.

The study was extended with simple and contrasted brain nuclear magnetic resonance imaging (MRI) in order to characterize the lesion. The initial diagnostic impression was a metastatic lesion; therefore, extension studies were requested in order to search for possible primary neoplasm, which were negative. Anti-edema management was started with intravenous 8 $\mathrm{mg}$ of dexamethasone every $8 \mathrm{~h}$ and anticonvulsant management was managed with $500 \mathrm{mg}$ of levetiracetam every $12 \mathrm{~h}$.

The simple and contrasted MRI (Fig. 2) showed a lesion with well-defined intra-axial left frontal edges, isointense in T1 and T2 sequences, with homogeneous uptake of the contrast medium, without restriction to diffusion, associated with large perilesional edema, with deviation of the midline to the right and subfalcine herniation (Fig. 2).

The patient underwent surgical intervention with prior skull CT-guided surgical site marking (Fig. 3). The procedure 


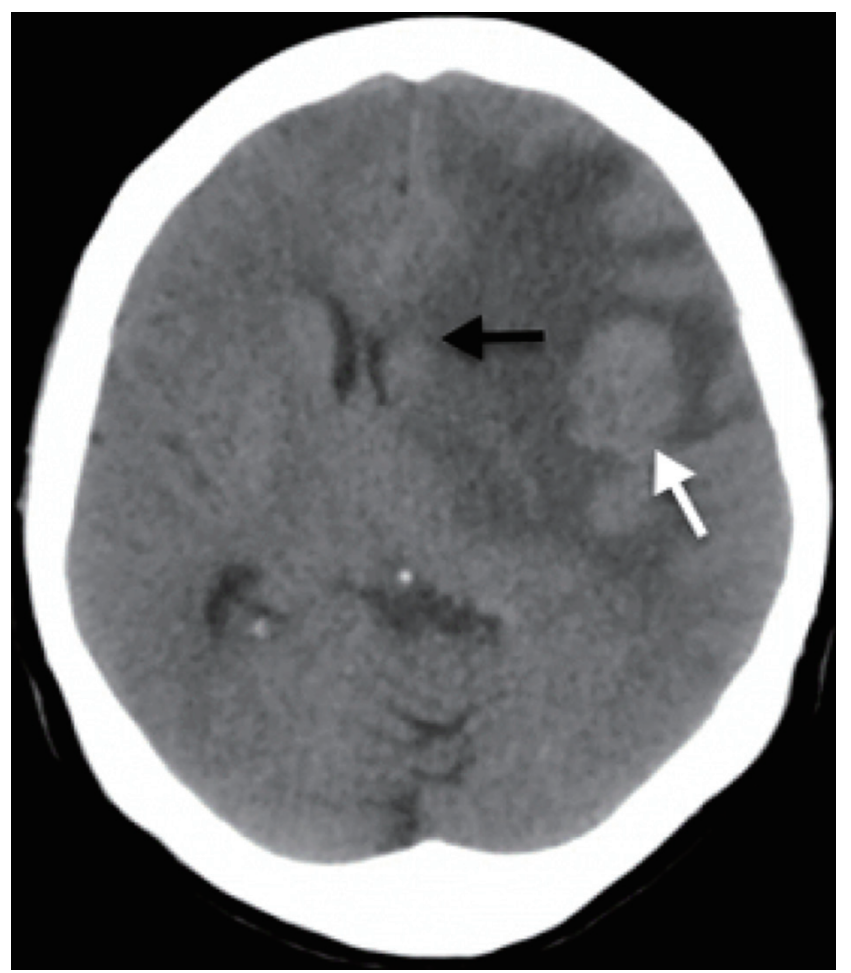

Figure 1. Simple skull CT scan. A rounded lesion is seen in the middle portion of the subcortical cortical area in the left frontal lobe (white arrow) with vasogenic edema that together, generate a mass effect with deviation of the midline to the right, compression of lateral ventricles and subfalcine herniation (black arrow). CT: computed tomography.

was carried out under general anesthesia, with a left pterional craniotomy. Through intraoperative ultrasonographic guid- ance, the tumor lesion was identified and delimited in the left inferior frontal opercular subcortical gyrus. A corticotomy was carried out through the fissure and after $0.5 \mathrm{~cm}$ of depth, the lesion was identified. A blunt dissection was conducted through the capsular plane and progressive devascularization was carried out, subsequently achieving complete exeresis of the lesion. Intraoperatively, a solid encapsulated violetcolored tumor was observed, highly suggestive of meningioma.

During the postoperative follow-up, there were no motor or sensory focal neurological signs. Motor aphasia was evident, which resolved after the first $48 \mathrm{~h}$. A control tomography was carried out, with evidence of postsurgical changes and decrease in vasogenic edema. The pathology report informs a lesion compatible with transitional meningioma, World Health Organization (WHO) grade I. The patient was discharged without sequels of neurological deficit, with the indication to continue the outpatient follow-up. In her first outpatient post-operative follow-up after 2 weeks, she remained asymptomatic, without neurological deficit and a healthy surgical wound.

\section{Discussion}

Embryologically, meningiomas originate in the cerebral meninges, specifically in the arachnoid meningothelial cells [1], constituting the most common extra-axial lesion in the skull $[2,3]$ and one of the most frequent primary neoplasms of the central nervous system (CNS) $[4,9]$. They normally develop between the fifth and sixth decade of life, being more frequent in women (60-80\% of cases) [2,8]. This significant difference has been mainly related to hormonal factors, especially to the presence of progesterone [2]. Specifically, intraparenchymal

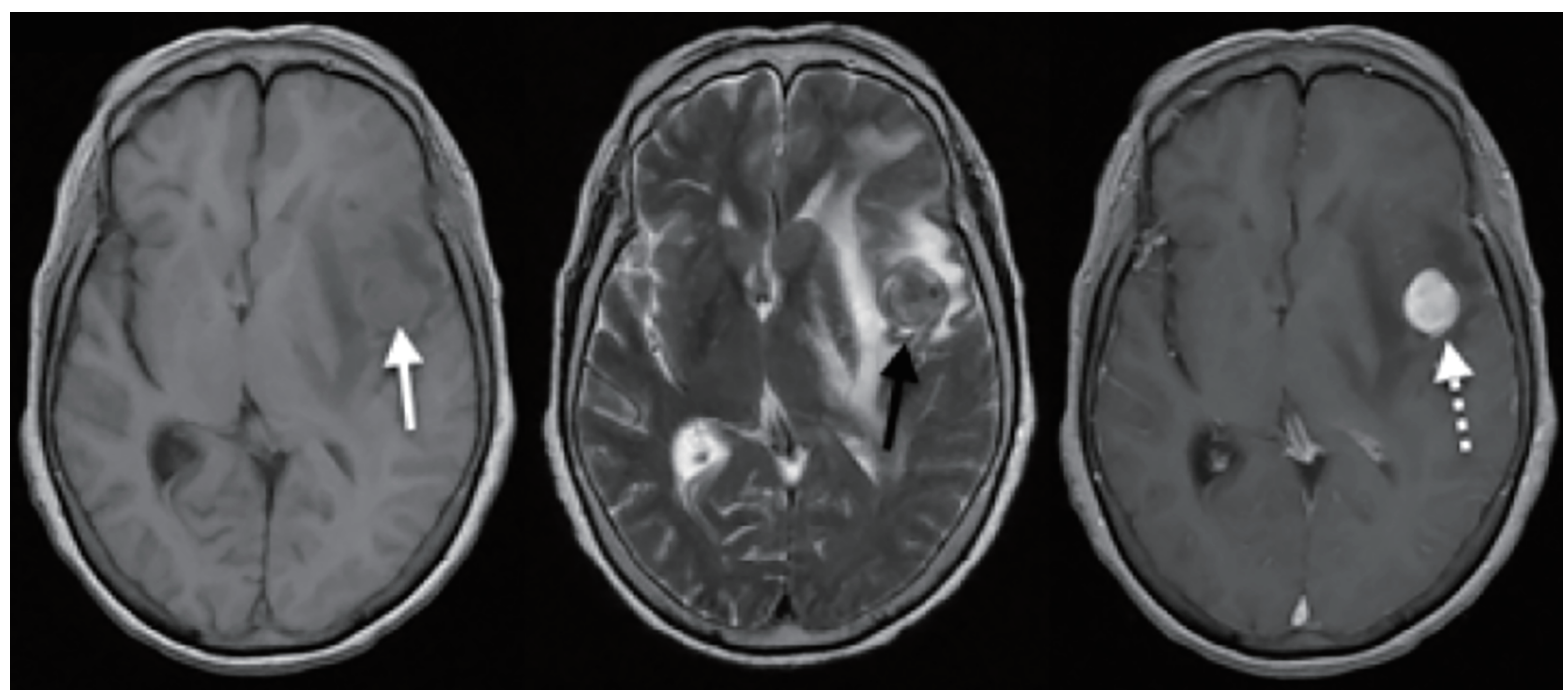

Figure 2. Simple and contrasted brain MRI. A homogeneous isointense rounded lesion in $\mathrm{T} 1$ is seen in the medical subcortical cortical region of the left frontal lobe (white arrow), with associated vasogenic edema and compressive effect on the lateral ventricles, with deviation of the midline to the right. For the T2 sequence, heterogeneous densities resembled its interior with a hypointense component and the extent of digitiform edema is seen in better detail (black arrow). After administration of the contrast medium, a homogeneous uptake of the entire lesion is observed (white dashed arrow), highlighting its rounded and circumscribed shape. MRI: magnetic resonance imaging. 


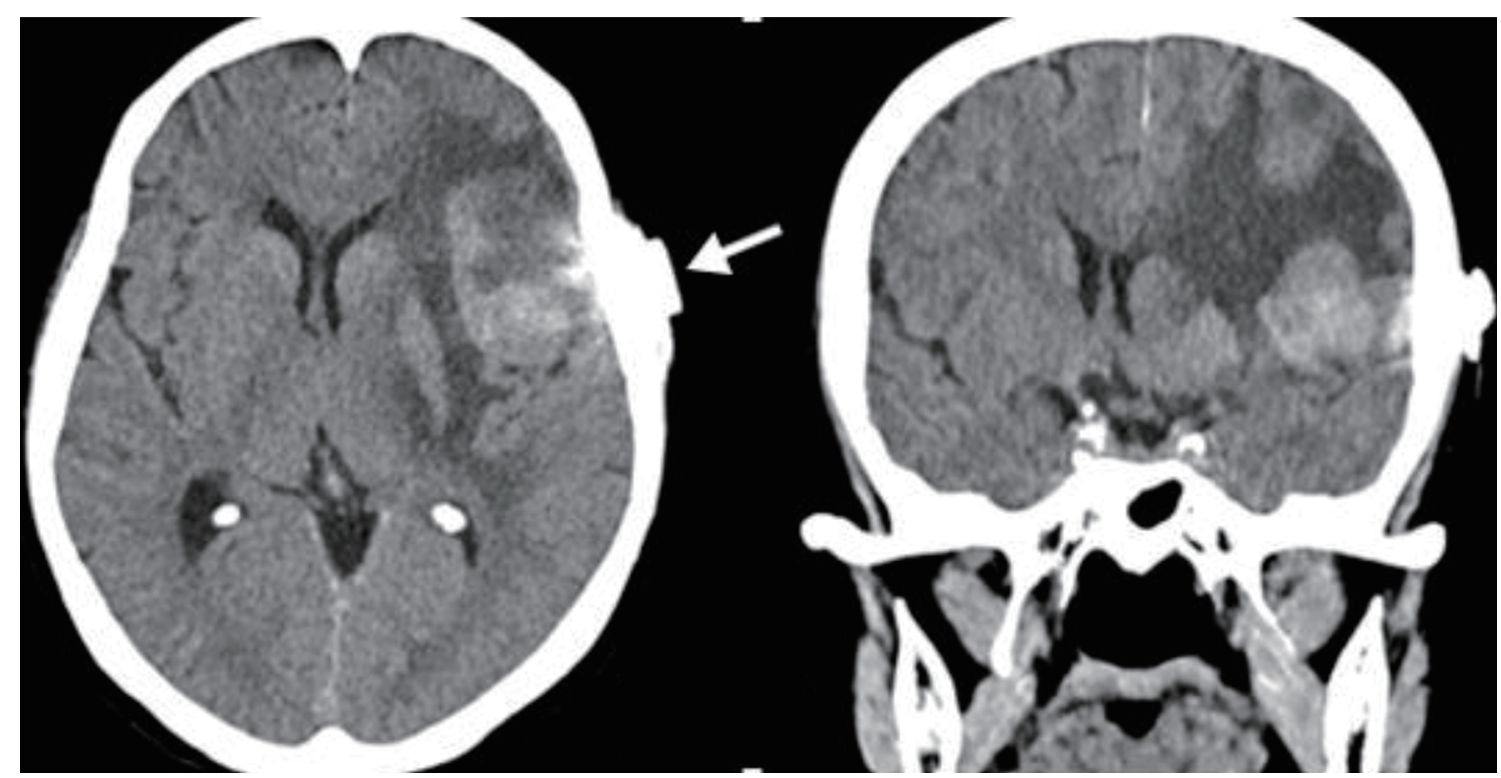

Figure 3. Simple skull tomography marking. An axial and coronal cut is evident through marking by marker (white arrow), to define an entry site with a direct path towards the lesion.

meningiomas have a different epidemiological pattern, they occur more commonly in men and in a younger population. [2]. On the other hand, in the pediatric population they represent approximately $1-2 \%$ of CNS tumors [2, 4, 6-8], with an annual incidence in the USA of 0.13 per 100,000 inhabitants [4]. Paradoxically, the presence of intraparenchymal meningiomas has been found mostly in pediatric patients, with more extensive patient cohorts and case reports. [3, 4, 8].

The pathophysiology of intraparenchymal meningiomas is not fully understood. It is considered that they can originate from ectopic meningothelial cells in the stroma of the choroid plexus or the pia mater, which can lead to intraventricular meningiomas, due to alteration in cell migration $[1,3]$. It has also been associated with pia mater and arachnoid stromal cells that accompany perforating blood vessels, which deepen from the cerebral cortex and between the sulci in some cases $[1-3,5,7$, 8], as well as to specific locations such as the Sylvian fissure or the Virchow-Robins spaces along the branches of the middle cerebral artery $[6,8]$. Perivascular meningothelial cells and especially, their proliferation, have been analyzed, determining that there might be an abnormal production of growth factors or loss of inhibitory factors, such as the neurofibromin 2 (NF2) gene inactivator, which are conditions that lead to meningiomas formation [3].

This category groups meningiomas without dural adhesion and completely surrounded by brain tissue [7]. Different locations have been described as intraventricular regarding the Sylvian fissure, pineal [1-3, 5-7] and lobar regions, being the frontal lobe predominant [1]. Cushing and Eisenhardt carried out a classification, grouping the meningiomas without dural adhesion depending on their location: 1) choroid plexus tumors; 2) choroidal tissue tumors; 3) deep Sylvian psammomeningiomas; 4) extracerebellar psammomeningiomas [2, $6,8]$. For those of supratentorial location, the following classification was designated: 1) intraventricular meningiomas; 2) pineal region meningiomas; 3) deep Sylvian meningiomas; 4) intraparenchymal or subcortical meningiomas; 5) others [2, 8], which together are more common than infratentorial ones [2]. Regarding the location in the posterior fossa, the following classification was designated: 1) meningiomas originating from the choroid plexus of the fourth ventricle; 2) meningiomas in the inferior choroid tissue; 3) meningiomas located in the cisterna magna; 4) intraparenchymal meningiomas [2]. There are case reports with even more atypical locations, such as the basal ganglia [6].

Imaging analysis of this type of neoplasms, in both tomography and magnetic resonance imaging, is a challenge [6] because pre-surgical diagnosis rarely leans towards a meningioma. The absence of dural adhesion and its similarity to other intraaxial lesions makes the differential diagnosis challenging [3].

Differential presurgical diagnoses are described in literature as: high-grade gliomas, oligodendrogliomas, oligoastrocytomas, ependymomas, cavernous angiomas, brain metastases, sarcomatous lesions, malignant lymphomas, dysembryoplastic neuroepithelial tumor and granulomas [1, 2, 4, 6-8]. When it has been associated with vascular lesions as a differential diagnosis, the use of angiography has been proposed as an additional diagnostic aid $[1,8]$.

Certain imaging features have been determined after the administration of the contrast medium, such as a solid lesion with homogeneous enhancement $[1,2]$. In the tomography, it is seen as a hyperdense lesion that in the MRI behaves isointense in T1 and hyperintense in T2 [2]. In addition, it tends to present great surrounding vasogenic edema [2, 4]; punctually in this case, a greater probability of malignant transformation is described [6].

In some cases, there might be imaging findings in atypical MRIs, such as a non-homogeneous enhancement with the contrast medium, which may be absent in up to $30 \%$ of both typical and intraparenchymal meningiomas [2], calcifications 
or cystic components [2]. Cysts in meningiomas occur infrequently in adults with a percentage of 1.6 to $7 \%$, which increases in the pediatric population to more than $10 \%[2,4]$ and increases significantly when it comes to intraparenchymal meningiomas [2]. The cystic component of these tumors can be divided into two: intratumor cysts and extratumor or arachnoid cysts. Regarding this, there are several theories of its origin: those intratumoral, which are presumed to be secondary to necrosis or tumor degeneration, while the peripheral ones are presumed to have originated in peripheral tumor degeneration or previously formed arachnoid cysts.

Other theories assume that cysts are the result of fluid secretion directly from the tumor cells, the absorption of tumor hemorrhages, or cerebrospinal fluid present in the scar tissue adjacent to the meningioma [2]. Clinically, it has been found that the most common symptoms are seizures [1, 4, 7-9], followed by headache, hemiparesis [2, 5, 9] and sensory deficit [9]. In the case of the pediatric population, the second symptom in prevalence is the increase in head circumference [2]. A long asymptomatic period is not unusual $[8,9]$, making its timely diagnosis even more difficult.

Regarding histopathology in general, meningiomas are benign WHO grade I in 80 to $90 \%, 10$ to $15 \%$ are WHO grade II and 1 to $3 \%$ are WHO grade III, being the meningothelial variant the most common with $54 \%$ of prevalence $[1,2]$, while fibrous types correspond to $17 \%$ of typical meningiomas [2]. Regarding intraparenchymal meningiomas, it has been found that the most prevalent variants are fibrous ones with $44 \%$, doubling its prevalence in extra-axial meningiomas [1-3, 7 , 8]. However, other variants such as psammomatous types have also been documented [6].

Surgical management was indicated in most documented case reports and a total resection was achieved $[1,2,6]$, which especially in pediatric patients, significantly reduces the recurrence rate [8]. Even so, this type of procedure represents a challenge for the neurosurgeon, due to the fibrous nature of most tumors, making debulking difficult [4]. Some cases did not allow this type of resection, such as intraparenchymal meningiomas with involvement of the brainstem [1] or those that were closely related to the Sylvian fissure and the branches of the middle cerebral artery $[2,6]$, where subtotal resection was achieved and adjuvant management with radiotherapy was indicated [6].

\section{Conclusions}

Intraparenchymal meningiomas are a rare subtype of this neoplasm, which, due to their formation and location, are far from the definition of a tumor dependent on the cerebral meninges. This means that, together with its low prevalence, it is a presurgical diagnostic challenge. The joint analysis of case reports documented so far and the one we present here, allows us to find clinical, imaging and histological similarities that highlight the importance of considering intra-axial or intraparenchymal meningiomas as an important differential diagnosis in the study of the neoplastic and vascular lesions of the central nervous system.

\section{Acknowledgments}

None to declare.

\section{Financial Disclosure}

None to declare.

\section{Conflict of Interest}

None to declare.

\section{Informed Consent}

The informed consent was obtained.

\section{Author Contributions}

Jaime Arias and Raul Ramirez-Grueso contributed to the conception, design and critical review of the paper content. Jorge Amortegui-Beltran, Sergio Patino-Ladino, Jose L. Rios, Lina Estrada-Duque contributed to the data collection, analysis, interpretation, and paper elaboration.

\section{Data Availability}

The authors declare that data supporting findings reported on this study are available within the article.

\section{References}

1. Jadik S, Stan AC, Dietrich U, Pietila TA, Elsharkawy AE. Intraparenchymal meningioma mimicking cavernous malformation: a case report and review of the literature. J Med Case Rep. 2014;8:467.

2. Ohba S, Abe M, Hasegawa M, Hirose Y. Intraparenchymal meningioma: clinical, radiologic, and histologic review. World Neurosurg. 2016;92:23-30.

3. Tekkok IH, Cinel L, Zorludemir S. Intraparenchymal meningioma. J Clin Neurosci. 2005;12(5):605-608.

4. Larrew T, Eskandari R. Pediatric intraparenchymal meningioma: case report and comparative review. Pediatr Neurosurg. 2016;51(2):83-86.

5. Matsuda R, Shida Y, Nakamura M. Intraparenchymal meningioma in the basal ganglia. World Neurosurg. 2019; $128: 186-188$.

6. Samson Sujit Kumar G, Rajshekhar V. Deep sylvian meningioma: a case report and review of literature. Childs Nerv Syst. 2009;25(1):129-132.

7. Karadereler S, Aker F, Berkman Z. Intraparenchymal meningioma in a child. Case report and review of the lit- 
erature. J Neurosurg. 2004;101(1 Suppl):112-115.

8. Shimbo D, Kato T, Takeda M, Ikeda H. Intraparenchymal meningioma in a child. Neurol Med Chir (Tokyo). 2011;51(11):793-797.
9. Wang DJ, Zheng MZ, Gong Y, Xie Q, Wang Y, Cheng HX, Mao Y, et al. Papillary meningioma: clinical and histopathological observations. Int J Clin Exp Pathol. 2013;6(5):878-888. 